\title{
$\beta$-cell function prior to liver transplantation contributes to post-operative diabetes
}

\author{
TATSUKI ICHIKAWA ${ }^{1,2}$, NAOTA TAURA ${ }^{1}$, HISAMITSU MIYAAKI ${ }^{1}$, SATOSHI MIUMA $^{1}$, \\ HIDETAKA SHIBATA ${ }^{1}$, TAKUYA HONDA ${ }^{1}$, MASAAKI HIDAKA ${ }^{3}$, AKIHIKO SOYAMA ${ }^{3}$, \\ MITSUHISA TAKATSUKI ${ }^{3}$, SUSUMU EGUCHI $^{3}$ and KAZUHIKO NAKAO ${ }^{1}$ \\ ${ }^{1}$ Department of Gastroenterology and Hepatology, Graduate School of Biomedical Sciences, \\ Nagasaki University, Nagasaki 852-8501; ${ }^{2}$ Department of Gastroenterology, Nagasaki Harbor \\ Medical Center, Nagasaki 850-8555; ${ }^{3}$ Department of Transplantation and Digestive Surgery, \\ Graduate School of Biomedical Sciences, Nagasaki University, Nagasaki 852-8501, Japan
}

Received May 23, 2016; Accepted September 7, 2016

DOI: $10.3892 /$ br.2016.788

\begin{abstract}
Liver cirrhosis and diabetes mellitus (DM) are closely associated. The present study aimed to determine whether liver transplantation (LT) may prevent/cure DM in patients with cirrhosis and whether the degree of glucose tolerance prior to transplantation is associated with the onset of DM after transplantation. Seventy-three patients who received a living donor LT at Nagasaki University Hospital (Nagasaki, Japan) between November 2005 and December 2012 were recruited. Among them, patients were considered diabetic if they had been prescribed diabetes medications or had impaired glucose tolerance, as evidenced by an oral glucose tolerance test (OGTT). Patients were followed up until December 31, 2013 to evaluate glucose tolerance. Patients who had developed
\end{abstract}

Correspondence to: Dr Tatsuki Ichikawa, Department of Gastroenterology, Nagasaki Harbor Medical Center, 6-39 Shinchi, Nagasaki 850-8555, Japan

E-mail: ichikawa@nagasaki-u.ac.jp

Abbreviations: LC, liver cirrhosis; T2DM, type 2 diabetes mellitus; HCC, hepatocellular carcinoma; HD, hepatogenous diabetes; BMI, body mass index; LT, liver transplantation; NODAT, new-onset diabetes after transplantation; LDLT, living donor liver transplantation; OGTT, oral glucose tolerance test; FPG, fasting plasma glucose; HbA1c, hemoglobin A1c; GA, glycated albumin; POY, post-operative year; $\mathrm{HCV}$, hepatitis $\mathrm{C}$ virus; $\mathrm{Cr}$, creatinine; $\mathrm{TG}$, triglyceride; TC, total cholesterol; HDL-C, high-density lipoprotein cholesterol; LDL-C, low-density lipoprotein cholesterol; IGT, impaired glucose tolerance; OGIS, oral glucose sensitivity; WBISI, whole-body insulin sensitivity index; MCR, metabolic clearance rate; HOMA-R, homeostasis model assessment of insulin resistance; HOMA-ISI, homeostasis model assessment insulin of sensitivity index; HOMA- $\beta$, homeostasis model assessment of insulin secretion; BCF, $\beta$-cell function; I. I., insulinogenic index; MELD, Model for End-Stage Liver Disease; CYA, cyclosporine A; TAC, tacrolimus

Key words: $\beta$-cell function, insulin sensitivity, liver transplantation, diabetes mellitus
DM 2 years after transplantation were found to be older and the incidence of diabetes prior to transplantation $(n=73)$ was higher than in those who did not. Multivariate analysis revealed that $\mathrm{DM}$ requiring treatment prior to transplantation was the only independent factor for DM developed at 2 years after transplantation. OGTT results showed that in patients with poor insulin sensitivity indices prior to transplantation $(n=45)$, improvements were seen at 2 years after transplantation, while $\beta$-cell function and insulinogenic index had decreased, which may have been the cause of DM after transplantation. In conclusion, the pre-operative $\beta$-cell function determined by an OGTT may be a useful predictive tool for the recurrence of DM after LT.

\section{Introduction}

Liver cirrhosis (LC) and diabetes mellitus (DM) are clinical conditions that are closely associated. Type 2 DM (T2DM) patients are at risk of liver disease, hepatocellular carcinoma (HCC) and death from hepatobiliary disease $(1,2)$. The risk of chronic liver disease and cirrhosis is also increased in the presence of DM (3). Diabetes with concurrent cirrhosis is called hepatogenous diabetes (HD) and differs from T2DM. HD is characterized by late onset of cirrhosis, negative family history for DM, low body mass index (BMI), reduced frequency of diabetic complications (retinopathy, cardiovascular disease and renal disease) and marked insulin resistance (3). However, in terms of diagnosis, the differentiation between T2DM and HD is difficult. HD and insulin sensitivity may resolve after liver transplantation (LT), while decreased insulin secretion is not attenuated after LT (4). Therefore, it is important to determine whether diabetes, particularly HD, may be prevented or cured by LT in patients with LC.

New-onset diabetes after transplantation (NODAT) is a serious metabolic complication with a reported incidence of $15-30 \%$ in patients receiving LT (5). Amongst previous studies, the definition and reported incidence of NODAT shows a wide variation owing to the heterogeneity in study design, variability of corticosteroid dose and immunosuppression protocols, as well as definition of post-transplant 
DM $(5,6)$. NODAT may bear an increased risk of morbidity and mortality after solid organ transplantation, particularly in the long term (7). To date, the pathophysiology of NODAT has not been clearly defined and may resemble that of T2DM, predominantly characterized by insulin resistance and/or defective insulin secretion (7). The development of NODAT has been attributed to multiple risk factors (7). Therefore, it is important to determine whether abnormal glucose tolerance (insulin resistance and/or insulin secretion) in patients without diabetes prior to LT may be associated with NODAT.

Patients with cirrhosis awaiting a living donor LT (LDLT) at Nagasaki University Hospital (Nagasaki, Japan) were subjected to oral glucose tolerance tests (OGTTs) in September 2005, prior to which they had not received any anti-diabetic treatments (insulin and/or oral medications). Blood glucose was evaluated on the basis of fasting plasma glucose (FPG), hemoglobin A1c (HbA1c) and glycoalbumin (GA) levels prior to and after LDLT. At post-operative year (POY) 2, corticosteroid therapy was discontinued and patients received low-dose calcineurin inhibitors; therefore, their glucose tolerance prior to LT and at POY2 was compared. In the present study, it was evaluated whether LT was able to resolve pre-existing DM.

\section{Patients and methods}

Patients. LC patients who were admitted for LDLT at Nagasaki University Hospital between November 2005 and December 2012 were recruited and followed up for complications and/or mortality until December 31, 2013. The inclusion criteria were as follows: First LDLT, adult (at least 20 years old), presence of chronic liver disease and blood glucose levels evaluated prior to LDLT. A total of 125 patients who underwent LDLT were assessed, of which 34 patients were excluded (Fig. 1); 18 patients died within 1 year after LT and 73 patients were diagnosed with diabetes after LT (Study 1). While liver biopsy is routinely performed at our clinic to detect hepatitis $\mathrm{C}$ virus (HCV)-associated complications after LT and liver dysfunction as well as other complications, the patients of the present study were instead subjected to a 75-g OGTT to confirm the diagnosis of diabetes. A total of 45 patients who underwent the 75-g OGTT after LT were enrolled in Study 2, while 28 patients who did not undergo 75 -g OGTT post-LT were excluded. Furthermore, 39 patients who underwent $75-\mathrm{g}$ OGTT prior to and subsequent to LT were enrolled in Study 3 , while 6 patients treated for diabetes prior to LT but did not undergo the 75-g OGTT prior to LT were excluded (Study 3). Informed consent was obtained from each patient included in the present study, and the study protocol conformed to the ethical guidelines of the 1975 Declaration of Helsinki, as evidenced by the approval by the Human Research Ethics Committee of Nagasaki University (Nagasaki, Japan).

Laboratory measurements. Laboratory data and anthropometric measurements were obtained for each subject prior to and after LT. The BMI was calculated as the weight in $\mathrm{kg}$ divided by the square of height in $\mathrm{m}$. Laboratory examinations included assessment of the white blood cell count, platelet count, prothrombin time, hemoglobin $(\mathrm{Hb})$, C-reactive protein, blood urea nitrogen, creatinine $(\mathrm{Cr})$, total protein, albumin,
125 patients received liver transplantation from Sept 2005 to Dec 2012

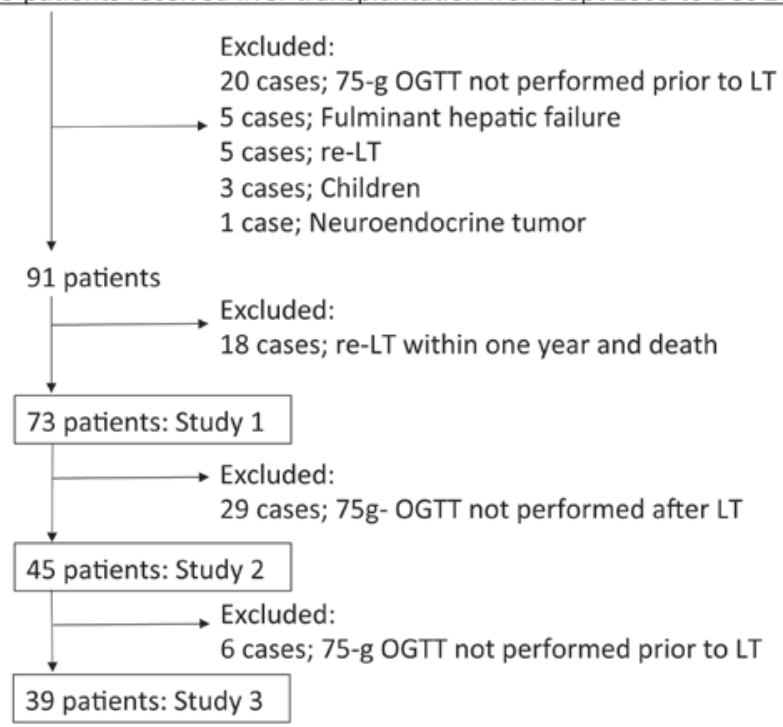

Figure 1. Flow diagram of enrolment of patients in the studies. OGTT, oral glucose tolerance test; LT, liver transplantation.

total bilirubin, alanine aminotransferase, $\gamma$-glutamyl transpeptidase, cholinesterase, triglyceride (TG), total cholesterol (TC), high-density lipoprotein cholesterol (HDL-C), low-density lipoprotein cholesterol (LDL-C), FPG, HbAlc and glycated albumin (GA) levels. HbA1c was measured using the method for patients prior to LDLT recommended by the Japan Diabetes Society (8).

Diagnosis of DM prior to and after LT. Patients were examined for the presence of DM 3 months prior to LT. DM was confirmed if i) the patient was prescribed at least one medication for DM or ii) had an FPG level of $\geq 126 \mathrm{mg} / \mathrm{dl}$. Patients without DM at admission underwent a 75-g OGTT and were diagnosed with DM if their FPG levels were $\geq 126 \mathrm{mg} / \mathrm{dl}$ or if their PG levels were $\geq 200 \mathrm{mg} / \mathrm{dl}$ at 120 post-prandial minutes. Patients with an FPG level of $\leq 110 \mathrm{mg} / \mathrm{dl}$ or $\leq 140 \mathrm{mg} / \mathrm{dl}$ at 120 post-prandial minutes were considered as having normal glucose tolerance (normal pattern), while those whose levels were above were categorized as having impaired glucose tolerance (IGT) and therefore diagnosed with DM.

Calculations of indices for insulin sensitivity and $\beta$-cell function. The 75-g OGTT was performed after $10 \mathrm{~h}$ of overnight fasting. Venous blood samples were obtained at $0,30,60$, 90 and 120 post-prandial minutes for determination of PG and serum insulin levels. Insulin sensitivity was estimated from PG, and insulin values were determined according to methods described by Mari et al (9) [oral glucose insulin sensitivity (OGIS)], Matsuda and DeFronzo (10) [whole body insulin sensitivity index (WBISI)], Stumvoll et al (11) [metabolic clearance rate of glucose (MCR)], homeostasis model assessment of insulin resistance [HOMA-R; PG at time 0 $(\mathrm{mg} / \mathrm{dl}) \mathrm{x}$ insulin at time $0(\mu \mathrm{U} / \mathrm{ml}) / 405](12)$, and homeostasis model assessment of insulin sensitivity index (HOMA-ISI; 1/HOMA-R) (12). $\beta$-cell function (BCF) was estimated from PG and insulin values according to methods described by Stancáková et al (13), Stumvoll et al [first-phase insulin release 
Table I. Association between DM requiring treatment at two years after LT and clinical factors existing prior to LT (Study 1).

\begin{tabular}{lccc}
\hline Parameter & DM+ $(\mathrm{n}=27)$ & DM- $(\mathrm{n}=46)$ & P-value \\
\hline Age (years) & $60 \pm 7.3$ & $55.2 \pm 9.3$ & 0.020 \\
Gender female/male (n) & $12 / 15$ & $22 / 24$ & $\mathrm{NS}$ \\
BMI (kg/m ${ }^{2}$ & $24.6 \pm 4.4$ & $24.4 \pm 3.9$ & $\mathrm{NS}$ \\
MELD & $13.3 \pm 7.4$ & $13.6 \pm 8.2$ & $\mathrm{NS}$ \\
Cr $(\mathrm{mg} / \mathrm{dl})$ & $1.05 \pm 0.85$ & $0.8 \pm 0.29$ & 0.0733 \\
TG (mg/dl) & $92 \pm 48.9$ & $69 \pm 44$ & 0.0533 \\
TC (mg/dl) & $122.2 \pm 39.9$ & $124 \pm 52.6$ & $\mathrm{NS}$ \\
DM +/- (n) & $18 / 9$ & $4 / 42$ & $<0.0001$ \\
DM+DM pattern +/- (n) & $25 / 2$ & $26 / 20$ & 0.0013 \\
HCV +/- (n) & $17 / 10$ & $19 / 27$ & 0.0913 \\
HCC +/- (n) & $18 / 9$ & $9 / 25$ & 0.0923
\end{tabular}

Values for Pre-LT clinical parameters are expressed as the mean \pm standard deviation and were subjected to the t-test. Patient numbers for gender, DM, DM+DM pattern, HCV and HCC were subjected to the $\chi^{2}$ test. DM, patients treated for diabetes prior to LT; DM pattern, patients with diabetes pattern as per 75-g oral glucose tolerance test results. HCV, hepatitis C virus; DM, diabetes mellitus; LT, liver transplantation; HCC, hepatocellular carcinoma; BMI, body mass index; MELD, Model for End-Stage Liver Disease score; Cr, creatinine; TG, triglyceride; TC, total cholesterol; NS, no significance $(\mathrm{P}>0.01)$.

(Phase 1)] and second-phase insulin release (Phase 2)] (11), homeostasis model assessment of insulin secretion [HOMA- $\beta$; insulin at time $0(\mu \mathrm{U} / \mathrm{ml}) \times 360 / \mathrm{PG}$ at time $0(\mathrm{mg} / \mathrm{dl})-63]$ and the insulinogenic index [I. I.; (insulin at $30 \mathrm{~min}$ - insulin at time 0$) /(\mathrm{PG}$ at $30 \mathrm{~min}-\mathrm{PG}$ at time 0$)](13)$.

Statistical analysis. Data were analyzed using the StatView 5.0 software (SAS Institute, Inc., Cary, NC, USA). Laboratory result variables were compared between DM and DM-free patients using t-tests and $\chi^{2}$ tests. Values for Pre-LT clinical parameters are expressed as the mean \pm standard deviation and were subjected to the t-test. Patient numbers for gender, DM, $\mathrm{DM}+\mathrm{DM}$ pattern, $\mathrm{HCV}$ and HCC were subjected to the $\chi^{2}$ test. Uni- and multivariate analyses were performed by logistic regression. $\mathrm{P}<0.05$ was considered to indicate a statistically significant difference.

\section{Results}

None of the patients was subjected to assessment of hepatic steatosis by ultrasonography or computed tomography prior to LT. Patients with fasting and post-prandial PG levels $\geq 250 \mathrm{mg} / \mathrm{dl}$ were treated with insulin or oral medication prior to and after LT. DM treatment was considered successful if blood glucose levels were maintained $<250 \mathrm{mg} / \mathrm{dl}$. In Study 1, the association between diabetes requiring treatment at POY2 and clinical factors prior to LT was assessed in 73 patients (Table I). Prior to LT, 22 patients were treated for DM and at two years after LT, 27 patients were in treatment. Among the patients treated for DM prior to LT, nine did not require treatment for DM after LT, and among those not treated for DM prior to LT, four required treatment after LT. Patients with DM at POY2 were older at LT $(60 \pm 7.3$ vs. $55.2 \pm 9.3$ years; $\mathrm{P}=0.02)$, had a higher incidence of DM prior to LT and more frequently showed a DM+DM pattern than patients without DM at POY2. $\mathrm{Cr}$ and TG levels as well as the incidence of HCV-infection and HCC were not significantly different between the DM and non-DM groups. The total intake of immunosuppressant steroid after LT did not differ between the DM and non-DM groups. Next, factors contributing to DM after LT were evaluated (POY2; Table II). According to univariate analysis, age as well as DM requiring treatment prior to LT contributed to DM developed at POY2. According to multivariate analysis, DM requiring treatment prior to LT was the only contributing factor for DM after LT [odds ratio, 0.038; 95\% confidence interval (CI): 0.008-0.183; $\mathrm{P}<0.0001]$.

For analysis of clinical and metabolic differences prior to and following LT (POY2) (Study 2; Table III), 29 patients who had not undergone the 75-g OGTT after LT in Study 1 were excluded. The results of the 75-g OGTT showed normal glucose levels in three patients prior to LT and in 18 patients at POY2 $(\mathrm{P}=0.0003)$. The BMIs of these patients prior to LT and at POY2 did not differ. While GA had improved at POY2, HbA1c showed no change compared with that prior to LT. Furthermore, HDL, LDL and TG levels were elevated at POY2. The PG value at 120 post-prandial minutes determined prior to LT was lower than that at POY2 (Fig. 2A). However, serum insulin values $(\mu \mathrm{U} / \mathrm{ml})$ at $0,60,90$ and 120 post-prandial minutes determined prior to LT were lower than those at POY2 (Fig. 2B). The 75-g OGTT revealed that the poor insulin sensitivity indices prior to LT improved after LT; furthermore, insulin secretion indexes (BCF and HOMA-B) were significantly decreased, while the I. I. as well as Phase 1 and 2 showed no changes (Table III).

Next, clinical and metabolic factors were compared between patients with normal glucose tolerance at POY2 (normal group; $n=17$ ) and those with IGT and DM at POY2 (IGT+DM group; n=22) (Study 3; Table IV). From the cohort of Study 2, six patients who had not undergone the 75-g OGTT prior to LT were excluded. The HbA1c levels in the normal group were slightly lower than those in the IGT+DM group $(\mathrm{P}=0.06)$. Furthermore, $\mathrm{PG}$ and insulin values were not different between 
Table II. Uni- and multivariate analysis for factors associated with DM developed at post-operative year 2 compared with patients who did not develop DM after LT (Study 1).

\begin{tabular}{lccccccc}
\hline & \multicolumn{3}{c}{ Univariate } & & \multicolumn{3}{c}{ Multivariate } \\
\cline { 2 - 3 } Parameter & P-value & Odds ratio & $95 \% \mathrm{CI}$ & & P-value & Odds ratio & $95 \% \mathrm{CI}$ \\
\hline Age & 0.0321 & 0.931 & $0.873-0.994$ & & $\mathrm{NS}$ & & \\
$\mathrm{Cr}$ & 0.1252 & 0.393 & $0.119-1.298$ & & $\mathrm{NS}$ & & \\
$\mathrm{TG}$ & 0.0644 & 0.991 & $0.979-1.001$ & & $\mathrm{NS}$ & & \\
$\mathrm{DM}^{\mathrm{a}}$ & $<0.0001$ & 0.039 & $0.010-0.152$ & & $<0.0001$ & 0.038 & \\
$\mathrm{HCV}$ & 0.0712 & 2.42 & $0.909-6.416$ & & $\mathrm{NS}$ & & \\
$\mathrm{HCC}$ & 0.0833 & 2.38 & $0.886-6.398$ & & $\mathrm{NS}$ & & \\
\hline
\end{tabular}

${ }^{a} \mathrm{DM}$ requiring treatment prior to LT. Logistic regression analysis was performed for diabetes requiring treatment after LT. Contributing factors were selected in Table I. HCV, hepatitis C virus; DM, diabetes mellitus; LT, liver transplantation; HCC, hepatocellular carcinoma; Cr, creatinine; TG, triglyceride; TC, total cholesterol; CI, confidence interval; NS, no significance.

Table III. Clinical and metabolic differences prior to and after LT (Study 2).

\begin{tabular}{lccc}
\hline Parameter & Prior to LT & POY2 & P-value \\
\hline DM+IGT/Normal (n) & $42 / 3$ & $27 / 18$ & 0.0003 \\
BMI $\left(\mathrm{kg} / \mathrm{m}^{2}\right)$ & $24.7 \pm 16.1$ & $24.2 \pm 4.13$ & $\mathrm{NS}$ \\
HbAlc $(\%)$ & $4.86 \pm 1.46$ & $5.25 \pm 0.66$ & $\mathrm{NS}$ \\
GA $(\%)$ & $20.5 \pm 1.03$ & $16.2 \pm 2.22$ & 0.0021 \\
HDL $(\mathrm{mg} / \mathrm{dl})$ & $31.4 \pm 16.4$ & $50.2 \pm 20$ & $<0.0001$ \\
LDL $(\mathrm{mg} / \mathrm{dl})$ & $55.2 \pm 23.1$ & $97.5 \pm 39.3$ & $<0.0001$ \\
TG $(\mathrm{mg} / \mathrm{dl})$ & $78.5 \pm 49$ & $121.7 \pm 57.6$ & 0.0004 \\
MELD & $12.1 \pm 7.1$ & $7.7 \pm 7.9$ & 0.0072 \\
75-g OGTT (n) & 39 & 45 & \\
Insulin sensitivity & & & \\
HOMA-R & $3.66 \pm 2.26$ & $1.63 \pm 0.89$ & $<0.0001$ \\
WBISI & $3.14 \pm 2.37$ & $5.66 \pm 2.3$ & $<0.0001$ \\
OGIS & $366.1 \pm 76$ & $418 \pm 52.5$ & 0.0004 \\
HOMA-ISI & $0.41 \pm 0.33$ & $0.81 \pm 0.41$ & $<0.0001$ \\
MCR & $3.17 \pm 4.9$ & $6.99 \pm 1.85$ & $<0.0001$ \\
BCF indices & & & \\
BCF & $60.2 \pm 35.5$ & $41.8 \pm 27.3$ & 0.0081 \\
HOMA- $\beta$ & $233.6 \pm 178.4$ & $115.1 \pm 88.3$ & $<0.0001$ \\
Insulinogenic index & $3.4 \pm 4.6$ & $7.1 \pm 20.6$ & NS \\
Phase 1 & $1,235 \pm 704$ & $956 \pm 1,033$ & NS \\
Phase 2 & $300.1 \pm 203.1$ & $221.2 \pm 256.8$ & NS \\
\hline
\end{tabular}

Values are expressed as the mean \pm standard deviation. DM, diabetes mellitus; LT, liver transplantation; BMI, body mass index; $\mathrm{Hg}$, hemoglobin; MELD, Model for End-Stage Liver Disease score; HDL-C, high-density lipoprotein cholesterol; LDL-C, low-density lipoprotein cholesterol; TG, triglycerides; GA, glycated albumin; IGT, impaired glucose tolerance; OGIS, oral glucose sensitivity; WBISI, whole-body insulin sensitivity index; MCR, metabolic clearance rate; HOMA-R, homeostasis model assessment of insulin resistance; HOMA-ISI, homeostasis model assessment insulin of sensitivity index; HOMA- $\beta$, homeostasis model assessment of insulin secretion; BCF, $\beta$-cell function; POY, post-operative year; OGTT, oral glucose tolerance test; phase $1 / 2$, phase $1 / 2$ insulin release; NS, no significance. the normal and IGT+DM groups (Fig. 2C and D). However, the MCR of glucose, insulin sensitivity indices and BCF indices in the normal group were better than those in the IGT+DM group. Next, clinical and metabolic factors prior to LT were compared between patients with DM at POY2 (DM group; $n=10$ ) and normal patients or those with IGT at POY2 (Normal+IGT group; $\mathrm{n}=29$ ) (Study 3, Table V). The BMI values before LT and at POY2 were different between the Normal+IGT and DM groups. PG values in the Normal+IGT group at the time-point 0 were lower than those in the DM group (Fig. 2E). However, serum insulin values in the Normal+IGT group at all times were lower than those in the DM group (Fig. 2F). According to the results of the 75-g OGTT, the MCR values in the Normal+IGT group were lower than those in the DM group. However, in the Normal+IGT group, BCF indices, excluding the I. I., were better than those in the DM group. Finally, factors contributing to normal glucose tolerance after $\mathrm{LT}$ at POY2 were assessed (Table VI). According to univariate analysis, BCF was the only contributing factor to normal glucose tolerance after LT (POY2), while it was not an independent factor associated with normal glucose tolerance after LT according to multivariate analysis. In addition, the factors contributing to a DM pattern after LT (POY2) were assessed (Table VI). According to univariate analysis, the BCF, MCR and I. I. were factors contributing to a DM pattern after LT (POY2), among which I. I. was revealed to be an independent factor associated with DM after LT by multivariate analysis (odds ratio, 0.451; 95\% CI: 0.299-0.888; $\mathrm{P}<0.02$ ).

In Study 3, 20 out of 39 patients underwent 75-g OGTT at POY2 and POY5 and 5 out of these 20 patients underwent 75-g OGTT at POY2, POY5 and POY6 (Table VII). The incidence of DM and IGT did not differ between POY2, POY5 and POY6. In addition, the BMI, HbAlc levels and the stage of liver fibrosis did not differ between POY2, POY5 and POY6. Of note, GA levels at POY5 were found to be slightly increased compared to those at POY2 $(\mathrm{P}=0.06)$. With regard to the $75-\mathrm{g}$ OGTT results, $\mathrm{PG}$ levels at 120 post-prandial minutes at POY6 were significantly higher than those at POY5 (Fig. 2G) and insulin levels at 120 post-prandial minutes at POY2 were significantly lower than those at POY5 and POY6 (Fig. 2H). 
A

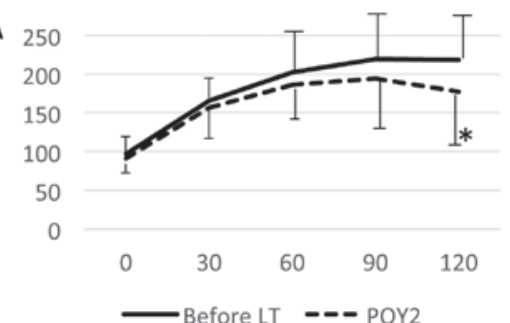

C

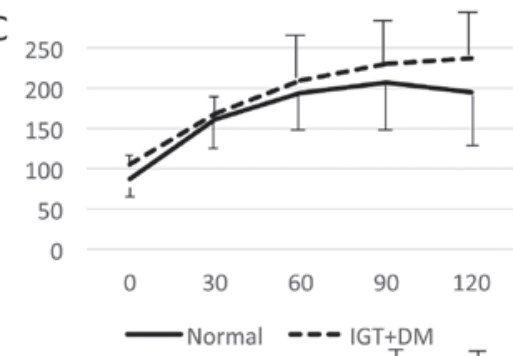

E

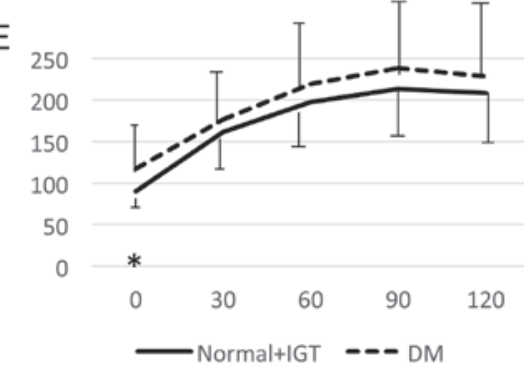

G

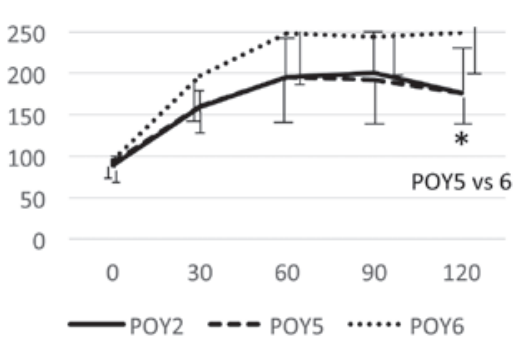

$B_{200}$

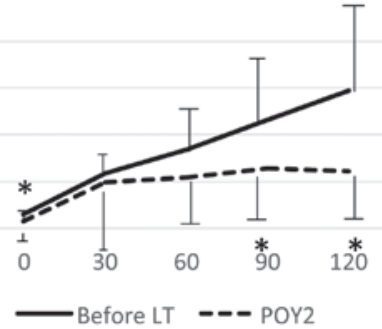

$D_{200}$

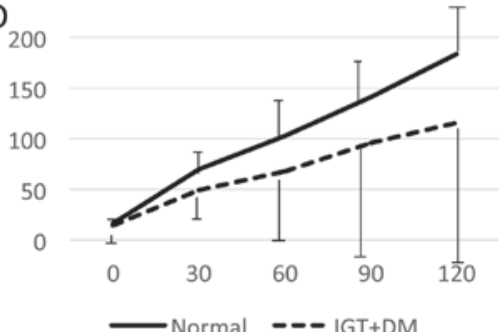

$\mathrm{F}_{200}$

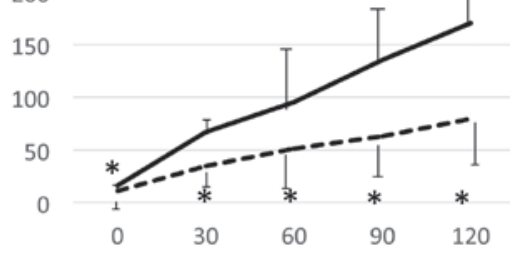

- Norma+|GT| $---D M$

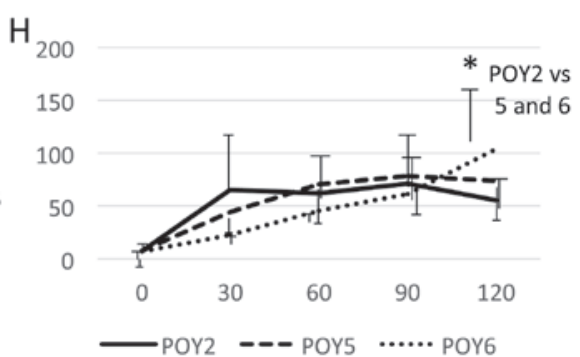

Figure 2. Patients' PG and insulin profiles obtained by 75 -g OGTT. The Y-axis shows PG values in $\mathrm{mg} / \mathrm{dl}$ or insulin in $\mu \mathrm{U} / \mathrm{ml}$, and the X-axis shows the post-prandial time (0, 30, 60, 90 and $120 \mathrm{~min}$ ). Comparison of (A) PG and (B) insulin levels before LT and at POY2 (Study 2). Comparison of (C) PG and (D) insulin levels before LT between the normal glucose tolerance after LT group and the IGT+DM after LT group (Study 3). Comparison of (E) PG and (F) insulin levels before LT between the normal+IGT after LT group and the DM after LT group (Study 3). Comparison of (G) PG and (H) insulin levels obtained at POY2, POY5 and POY6 (Study 3). Statistical analysis was performed between groups using the t-test. Values are expressed as the mean + or - the standard deviation. "P<0.05. POY, post-operative year, OGTT, oral glucose tolerance test; DM, diabetes mellitus; PG, plasma glucose; LT, liver transplantation; IGT, impaired glucose tolerance.

Furthermore, MCR at POY6 was significantly lower than that at POY2 and OGIS at POY2 was significantly higher than that at POY5. In addition, the I. I. at POY6 was higher than that at POY2 and POY5. Moreover, Phase $1 / 2$ at POY6 was lower than at POY2 and -5 .

\section{Discussion}

In the first part of the present study, clinical factors associated with DM after LT (POY2) were assessed (Study 1; n=73). To assess the recurrence of DM after LT, patients with DM treated with medication prior to LT were selected. Next, the glucose metabolism prior to LT and at POY2 were assessed (Study 2; n=45). LT clearly improved the insulin sensitivity at POY2, while BCF and HOMA- $\beta$ were significantly decreased. The possibility of advanced insulin resistance was noted at POY5 and -6. Furthermore, for evaluating the association between glucose metabolism prior to and after LT in Study 3 $(n=39), 75$-g OGTT results obtained prior to LT and at POY2 were compared. I. I. as an indicator of BCF prior to LT was revealed to be an independent factor contributing to a DM pattern according to the 75-g OGTT at POY2. These results may indicate that insulin resistance was improved by LT, while persisting $\beta$-cell dysfunction may have caused DM after LT.

In Study 1, the association of clinical factors prior to LT with the recurrence of DM after LT (POY2) was assessed. It was revealed that patients with DM at POY2 were older at the time of LT and showed a higher incidence of DM before LT compared with those without DM at POY2. According to multivariate analysis, DM requiring treatment prior to LT was the only contributing factor for DM at POY2. When patients with cirrhotic livers required treatment for DM prior to LT, LT presumably did not resolve DM at POY2. It has been reported that DM prior to LT leads to increased mortality after 
Table IV. Comparison of clinical parameters prior to LT between the normal group and the IGT+DM group (Study 3).

\begin{tabular}{|c|c|c|c|}
\hline Parameter & $\begin{array}{c}\text { Normal } \\
(n=19)\end{array}$ & $\begin{array}{c}\mathrm{IGT}+\mathrm{DM} \\
(\mathrm{n}=26)\end{array}$ & P-value \\
\hline Age at LT (years) & $55.6 \pm 10.4$ & $57.3 \pm 8.16$ & NS \\
\hline $\operatorname{BMI}\left(\mathrm{kg} / \mathrm{m}^{2}\right)$ & $25.3 \pm 4.6$ & $24.3 \pm 3.6$ & NS \\
\hline Gender female/male (n) & $7 / 12$ & $11 / 15$ & NS \\
\hline MELD & $13.2 \pm 7.6$ & $11.3 \pm 6.8$ & NS \\
\hline $\mathrm{Cr}(\mathrm{mg} / \mathrm{dl})$ & $0.94 \pm 0.47$ & $0.75 \pm 0.24$ & NS \\
\hline $\mathrm{HCV}+/-(\mathrm{n})$ & $10 / 9$ & $15 / 11$ & NS \\
\hline $\mathrm{HCC}+/-(\mathrm{n})$ & $9 / 10$ & $13 / 13$ & NS \\
\hline $\mathrm{DM}+/-(\mathrm{n})$ & $6 / 13$ & $16 / 10$ & 0.0701 \\
\hline $\mathrm{DM} 2+/-(\mathrm{n})$ & $16 / 3$ & $26 / 0$ & 0.0612 \\
\hline HbA1c $(\%)$ & $4.38 \pm 0.54$ & $5.2 \pm 1.84$ & 0.0622 \\
\hline GA $(\%)$ & $18.8 \pm 3.35$ & $21.6 \pm 7.49$ & NS \\
\hline LDL (mg/dl) & $57.5 \pm 27$ & $53.5 \pm 20.1$ & NS \\
\hline HDL (mg/dl) & $29.7 \pm 15.2$ & $32.6 \pm 17.5$ & NS \\
\hline TG (mg/dl) & $65.6 \pm 31.8$ & $88.4 \pm 57.5$ & NS \\
\hline Stage & $0.68 \pm 0.89$ & $0.6 \pm 0.75$ & NS \\
\hline CYA/TAC (n) & $4 / 14$ & $8 / 18$ & NS \\
\hline \multicolumn{4}{|l|}{ Insulin sensitivity } \\
\hline HOMA-R & $3.5 \pm 1.7$ & $3.6 \pm 2.9$ & NS \\
\hline WBISI & $3 \pm 2.5$ & $3.8 \pm 3.4$ & NS \\
\hline OGIS & $377 \pm 84.6$ & $356 \pm 68.3$ & NS \\
\hline HOMA-ISI & $0.43 \pm 0.4$ & $0.39 \pm 0.27$ & NS \\
\hline MCR & $1.94 \pm 6.1$ & $5.56 \pm 4.4$ & 0.0202 \\
\hline \multicolumn{4}{|l|}{$\beta$-cell function } \\
\hline $\mathrm{BCF}$ & $99.7 \pm 56.8$ & $65.4 \pm 36.6$ & 0.0211 \\
\hline HOMA- $\beta$ & $277 \pm 143$ & $196 \pm 200$ & NS \\
\hline Insulinogenic index & $1.78 \pm 1.1$ & $4.9 \pm 5.8$ & 0.0313 \\
\hline Phase 1 & $1,447 \pm 663$ & $1,054 \pm 701$ & 0.0801 \\
\hline Phase 2 & $329 \pm 149$ & $277 \pm 239$ & NS \\
\hline
\end{tabular}

Values are expressed as the mean \pm standard deviation. DM, diabetes mellitus; DM2, treated DM+DM pattern by OGTT prior to LT; LT, liver transplantation; BMI, body mass index; $\mathrm{Hg}$, hemoglobin; MELD, Model for End-Stage Liver Disease score; Cr, creatinine; TG, triglyceride; TC, total cholesterol; HDL-C, high-density lipoprotein cholesterol; LDL-C, low-density lipoprotein cholesterol; TG, triglycerides; GA, glycated albumin; IGT, impaired glucose tolerance; OGIS, oral glucose sensitivity; WBISI, whole-body insulin sensitivity index; MCR, metabolic clearance rate; HOMA-R, homeostasis model assessment of insulin resistance; HOMA-ISI, homeostasis model assessment insulin of sensitivity index; HOMA- $\beta$, homeostasis model assessment of insulin secretion; BCF, $\beta$-cell function; CYA/TAC, cyclosporine $\mathrm{A} /$ tacrolimus (calcineurin inhibitors administered to patients at POY2); Stage, liver fibrosis score at liver biopsy at two years after LT; POY, post-operative year; OGTT, oral glucose tolerance test; phase $1 / 2$, phase $1 / 2$ insulin release; NS, no significance.

LT $(14,15)$. However, according to another study, DM was associated with adverse events and a long hospital stay without directly affecting the survival after LT (16). Other studies also concluded that DM prior to LT is not associated with
Table V. Comparison of clinical factors before LT between the normal+IGT group and DM group (Study 3).

\begin{tabular}{|c|c|c|c|}
\hline Parameter & $\begin{array}{l}\text { Normal+IGT } \\
\quad(n=29)\end{array}$ & $\begin{array}{c}\text { DM } \\
(n=10)\end{array}$ & P-value \\
\hline Age at LT (years) & $55.9 \pm 9.33$ & $58.1 \pm 8.7$ & NS \\
\hline $\operatorname{BMI}\left(\mathrm{kg} / \mathrm{m}^{2}\right)$ & $25.2 \pm 3.3$ & $22.5 \pm 2.9$ & 0.0210 \\
\hline Gender f/m (n) & $12 / 17$ & $3 / 7$ & NS \\
\hline MELD & $12.2 \pm 7.1$ & $9.2 \pm 5$ & NS \\
\hline $\mathrm{Cr}(\mathrm{mg} / \mathrm{dl})$ & $0.87 \pm 0.41$ & $0.72 \pm 0.16$ & NS \\
\hline $\mathrm{HCV}+/-(\mathrm{n})$ & $17 / 12$ & $6 / 4$ & NS \\
\hline $\mathrm{HCC}+/-(\mathrm{n})$ & $16 / 13$ & $6 / 4$ & NS \\
\hline $\mathrm{DM}+/-(\mathrm{n})$ & $27 / 2$ & $10 / 0$ & NS \\
\hline DM2 +/- (n) & $16 / 16$ & $4 / 6$ & NS \\
\hline HbA1c (\%) & $4.5 \pm 0.58$ & $5.1 \pm 2.3$ & 0.0612 \\
\hline GA $(\%)$ & $18.4 \pm 3.1$ & $24 \pm 9$ & NS \\
\hline $\mathrm{LDL}(\mathrm{mg} / \mathrm{dl})$ & $56.1 \pm 26.3$ & $57.3 \pm 8.4$ & NS \\
\hline HDL (mg/dl) & $31.3 \pm 17.3$ & $37 \pm 13.6$ & NS \\
\hline TG (mg/dl) & $72.7 \pm 36.2$ & $91.2 \pm 77$ & NS \\
\hline Stage & $0.6 \pm 0.8$ & $0.79 \pm 0.8$ & NS \\
\hline CYA/TAC (n) & $8 / 23$ & $5 / 9$ & NS \\
\hline \multicolumn{4}{|l|}{ Insulin sensitivity } \\
\hline HOMA-R & $3.7 \pm 1.7$ & $3.5 \pm 3.5$ & NS \\
\hline WBISI & $2.8 \pm 2.1$ & $4.2 \pm 2.9$ & NS \\
\hline OGIS & $364 \pm 72.3$ & $369 \pm 88.8$ & NS \\
\hline HOMA-ISI & $0.39 \pm 0.34$ & $0.47 \pm 0.3$ & NS \\
\hline MCR & $2.18 \pm 5.2$ & $6.05 \pm 2.1$ & 0.0211 \\
\hline \multicolumn{4}{|l|}{$\mathrm{BCF}$ indices } \\
\hline $\mathrm{BCF}$ & $68.6 \pm 34.9$ & $36 \pm 25.5$ & 0.0134 \\
\hline НОМА- $\beta$ & $270 \pm 183$ & $127 \pm 84$ & 0.0212 \\
\hline Insulinogenic index & $1.9 \pm 1.3$ & $7.7 \pm 7.6$ & 0.0003 \\
\hline Phase 1 & $1,416 \pm 625$ & $712 \pm 682$ & 0.0041 \\
\hline Phase 2 & $348 \pm 187$ & $168 \pm 194$ & 0.0101 \\
\hline
\end{tabular}

Values are expressed as the mean \pm standard deviation. DM, diabetes mellitus; f, female; m, male; LT, liver transplantation; BMI, body mass index; Hg, hemoglobin; MELD, Model for End-Stage Liver Disease score; $\mathrm{Cr}$, creatinine; TG, triglyceride; TC, total cholesterol; HDL-C, high-density lipoprotein cholesterol; LDL-C, low-density lipoprotein cholesterol; TG, triglycerides; GA, glycated albumin; IGT, impaired glucose tolerance; OGIS, oral glucose sensitivity; WBISI, whole-body insulin sensitivity index; MCR, metabolic clearance rate; HOMA-R, homeostasis model assessment of insulin resistance; HOMA-ISI, homeostasis model assessment insulin of sensitivity index; HOMA- $\beta$, homeostasis model assessment of insulin secretion; $\mathrm{BCF}, \beta$-cell function; CYA/TAC, cyclosporine A/tacrolimus (calcineurin inhibitors administered to patients at POY2); Stage, liver fibrosis score at liver biopsy at two years after LT; POY, post-operative year; phase $1 / 2$, phase $1 / 2$ insulin release; OGTT, oral glucose tolerance test; NS, no significance.

mortality after LT (17-19). These conflicting results may be due to different diagnostic criteria for DM used in the studies, and blood glucose was not measured objectively in all of the studies. Patients with DM treated with medication prior to LT 
Table VI. Uni- and multivariate analysis of the glucose metabolic status after liver transplantation (Study 3).

A, Normal vs. IGT+DM

\begin{tabular}{|c|c|c|c|c|c|c|}
\hline \multirow[b]{2}{*}{ Parameter } & \multicolumn{3}{|c|}{ Univariate } & \multicolumn{3}{|c|}{ Multivariate } \\
\hline & P-value & Odds ratio & $95 \% \mathrm{CI}$ & P-value & Odds ratio & $95 \%$ CI \\
\hline MCR & 0.1 & 0.976 & $0.786-1.045$ & NS & & \\
\hline $\mathrm{BCF}$ & 0.04 & 1.027 & $1.000-1.054$ & 0.07 & 1.034 & $0.997-1.072$ \\
\hline I. I. & 0.08 & 0.682 & $0.439-1.059$ & & & \\
\hline
\end{tabular}

B, Normal+IGT vs. DM

Univariate

\begin{tabular}{lcccccr}
\cline { 2 - 4 } Parameter & P-value & Odds ratio & $95 \%$ CI & & P-value & Odds ratio \\
\hline BMI & 0.03 & 1.322 & $1.020-1.626$ & & NS & \\
MCR & 0.04 & 0.753 & $0.571-0.989$ & & NS & \\
I. I. & 0.02 & 0.615 & $0.406-0.993$ & 0.02 & 0.451
\end{tabular}

I. I., insulinogenic index; MCR, metabolic clearance rate; BMI, body mass index; NS, no significance; CI confidence interval; IGT, impaired glucose tolerance; DM, diabetes mellitus.

Table VII. Changes in glucose metabolic status at POY2, POY5 and POY6 (Study 3).

\begin{tabular}{|c|c|c|c|c|}
\hline Parameter & POY2 (n=20) & POY $5(n=20)$ & POY6 (n=5) & P-value \\
\hline DM/IGT/Normal (n) & $7 / 6 / 7$ & $5 / 9 / 6$ & $3 / 1 / 1$ & NS \\
\hline BMI $\left(\mathrm{kg} / \mathrm{m}^{2}\right)$ & $24.3 \pm 3.14$ & $25.3 \pm 3.93$ & $24.3 \pm 3.14$ & NS \\
\hline HbA1c (\%) & $5.2 \pm 0.67$ & $5.07 \pm 0.65$ & $5.4 \pm 0.8$ & NS \\
\hline $\mathrm{GA}(\%)$ & $17.3 \pm 3.22$ & $14.5 \pm 2.33$ & $14.1 \pm 3.16$ & $0.0641^{\mathrm{a}}$ \\
\hline Stage & $0.55 \pm 0.83$ & $0.65 \pm 1.2$ & $1.2 \pm 0.83$ & NS \\
\hline \multicolumn{5}{|l|}{ Insulin sensitivity } \\
\hline HOMA-R & $1.61 \pm 0.96$ & $1.75 \pm 1.05$ & $1.6 \pm 0.89$ & NS \\
\hline WBISI & $5.2 \pm 1.82$ & $5 \pm 2.1$ & $4.8 \pm 1.74$ & NS \\
\hline OGIS & $428.4 \pm 48.7$ & $397.5 \pm 47$ & $407.4 \pm 81.1$ & $0.040^{\mathrm{a}}$ \\
\hline HOMA-ISI & $0.86 \pm 0.49$ & $0.8 \pm 0.48$ & $0.77 \pm 0.33$ & NS \\
\hline MCR & $7.1 \pm 1.1$ & $6.21 \pm 2.2$ & $4.52 \pm 3.1$ & $0.0922^{\mathrm{a}}, 0.0041^{\mathrm{b}}$ \\
\hline \multicolumn{5}{|l|}{$\mathrm{BCF}$ indices } \\
\hline $\mathrm{BCF}$ & $45.9 \pm 34.1$ & $46.1 \pm 22.3$ & $30.6 \pm 18.2$ & NS \\
\hline НОМА- $\beta$ & $111 \pm 65.2$ & $112.5 \pm 73.5$ & $100.8 \pm 62$ & NS \\
\hline Insulinogenic index & $3.9 \pm 6$ & $3.0 \pm 3.3$ & $12.9 \pm 12.9$ & $0.0211^{\mathrm{b}}, 0.0033^{\mathrm{c}}$ \\
\hline Phase 1 & $1138 \pm 1484$ & $877 \pm 572$ & $263 \pm 570$ & $0.0422^{\mathrm{c}}$ \\
\hline Phase 2 & $258 \pm 359$ & $196 \pm 147$ & $45 \pm 170$ & $0.0511^{\mathrm{c}}$ \\
\hline
\end{tabular}

${ }^{\text {aPOY2 }}$ vs. 5; ' $\mathrm{POY} 2$ vs. 6 ; ' ${ }^{\mathrm{POY}} 5$ vs. 6 . Values are expressed as the mean \pm standard deviation. DM, diabetes mellitus; BMI, body mass index; Hg, hemoglobin; GA, glycated albumin; IGT, impaired glucose tolerance; OGIS, oral glucose sensitivity; WBISI, whole-body insulin sensitivity index; MCR, metabolic clearance rate; HOMA-R, homeostasis model assessment of insulin resistance; HOMA-ISI, homeostasis model assessment insulin of sensitivity index; HOMA- $\beta$, homeostasis model assessment of insulin secretion; BCF, $\beta$-cell function; POY, post-operative year; phase 1/2, phase 1/2 insulin release; NS, no significance; Stage, liver fibrosis score at liver biopsy two years after liver transplantation.

continued to have metabolic disorders after LT and several of them even experienced cardiovascular events (7). The association between DM prior to LT and long-term prognosis after LT therefore requires to be further investigated. 
In the second module of the present study $(n=45)$, glucose metabolism prior to LT and at POY2 was assessed. The 75-g OGTT revealed that poor insulin sensitivity indices prior to LT improved at POY2, while I. I. and phase $1 / 2 \mathrm{did}$ not change at POY2. While the reasons for the decreases in BCF and HOMA- $\beta$ remain elusive, it is likely that the decrease of fasting insulin secretion at POY2 may have contributed to this. MCR at POY2 were found to decrease significantly at POY6, and OGIS at POY2 was also found to decrease significantly at POY5 (Study 3). LT can therefore improve insulin sensitivity at POY2 but cannot improve BCF at POY2. However, insulin sensitivity may progress to advanced insulin resistance after POY5. In Study 1, it was revealed that LT did not resolve DM, but according to Study 2, insulin sensitivity was improved by LT.

HD is characterized by insulin resistance (3). In accordance with the results of Mari et al (9), the present study found that LT improved insulin resistance but not insulin secretion. Impairment of insulin secretion begins even prior to the onset of DM (20). When patients with DM and cirrhosis are treated with anti-diabetic drugs, $\mathrm{BCF}$ is already at a progressive stage. Since in the present study, BCF in patients with DM treated with medication prior to LT was decreased, LT did not resolve DM at POY2 according to Study 1. Since insulin resistance was found to be elevated after POY5 in 20 patients in Study 3, it is recommended that patients should be followed up for a long period.

In Study $(n=39)$, the association between metabolic factors prior to and after LT 3 was evaluated by comparing 75-g OGTT results prior to LT and at POY2. In addition, further insulin sensitivity indices (OGIS, WBISI, MCR, HOMA-R and HOMA-ISI) and BCF indices (Phase 1, Phase 2, HOMA- $\beta$ and I. I.) were determined. The BCF was shown to be a contributing factor associated with normal glucose tolerance after LT (POY2) by univariate analysis, and the BCF index I. I. was the only independent factor associated with the DM pattern determined by the 75-g OGTT at POY2 according to multivariate analysis. It was therefore indicated that insulin resistance was improved by LT but BCF could not be resolved, which may have led to DM after LT.

It is known that LT decreases insulin secretion; however, the cause has remained elusive. Furthermore, it has been reported that denervation of the graft liver (21) and immunosuppressive therapy (22) do not cause impairment of BCF after LT. It is therefore suggested that the impairment of $\mathrm{BCF}$ is attributed to neural factors or increases in hormone levels secondary to immunosuppressive treatment (21). It has been reported that NODAT is associated with single nucleotide polymorphisms. The PNPLA3 gene polymorphism in recipients (23) and TCF7L2 gene polymorphism in the recipient and donor (24) have been implicated in the development of NODAT and even $\beta$-cell dysfunction $(25,26)$, and TCF7L2 was found to be associated with HD (27). It is therefore likely that patients with a genetic pre-disposition to $\beta$-cell dysfunction prior to LT develop diabetes or abnormal glucose tolerance after LT. The PNPLA3 gene is a known single-nucleotide polymorphism predictive of non-alcoholic steatohepatitis (23). In the present study, hepatic steatosis was not detected by imaging methods prior to LT, which should, however, be evaluated in future studies.
In conclusion, the present study indicated that insulin resistance was improved by LT and that diabetes at two years after LT is associated with $\beta$-cell dysfunction identified by a pre-existing diabetes requiring treatment prior to LT or a 75-g OGTT. In addition, loss of appetite is frequently seen in patients with advanced liver disease, and LT may improve liver function as well as appetite, which may contribute to NODAT; this association warrants elucidation in a future study.

\section{References}

1. Davis TME, Peters KE, Bruce DG and Davis WA: Prevalence, incidence, and prognosis of hepatobiliary disease in community-based patients with type 2 diabetes: The Fremantle Diabetes Study. J Clin Endocrinol Metab 97: 1581-1588, 2012.

2. El-Serag HB, Tran T and Everhart JE: Diabetes increases the risk of chronic liver disease and hepatocellular carcinoma. Gastroenterology 126: 460-468, 2004

3. Garcia-Compean D, Jaquez-Quintana JO, Gonzalez-Gonzalez JA and Maldonado-Garza H: Liver cirrhosis and diabetes: Risk factors, pathophysiology, clinical implications and management. World J Gastroenterol 15: 280-288, 2009.

4. Perseghin G, Mazzaferro V, Sereni LP, Regalia E, Benedini S, Bazzigaluppi E, Pulvirenti A, Leão AA, Calori G, Romito R, et al: Contribution of reduced insulin sensitivity and secretion to the pathogenesis of hepatogenous diabetes: Effect of liver transplantation. Hepatology 31: 694-703, 2000.

5. Marchetti P: New-onset diabetes after liver transplantation: From pathogenesis to management. Liver Transpl 11: 612-620, 2005.

6. Carey EJ, Aqel BA, Byrne TJ, Douglas DD, Rakela J, Vargas HE, Moss AA, Mulligan DC, Reddy KS and Chakkera HA: Pretransplant fasting glucose predicts new-onset diabetes after liver transplantation. J. Transplant 2012: 614781, 2012.

7. Dong M, Parsaik AK, Eberhardt NL, Basu A, Cosio FG and Kudva YC: Cellular and physiological mechanisms of new-onset diabetes mellitus after solid organ transplantation. Diabet Med 29: e1-e12, 2012.

8. Tominaga M, Makino H, Yoshino G, Kuwa K, Takei I, Aono Y, Hoshino T, Umemoto M, Shimatsu A, Sanke T, et al: Japanese standard reference material for JDS Lot 2 haemoglobin A1c. I: Comparison of Japan Diabetes Society-assigned values to those obtained by the Japanese and USA domestic standardization programmes and by the International Federation of Clinical Chemistry reference laboratories. Ann Clin Biochem 42: 41-46, 2005.

9. Mari A, Pacini G, Murphy E, Ludvik B and Nolan JJ: A model-based method for assessing insulin sensitivity from the oral glucose tolerance test. Diabetes Care 24: 539-548, 2001.

10. Matsuda M and DeFronzo RA: Insulin sensitivity indices obtained from oral glucose tolerance testing: Comparison with the euglycemic insulin clamp. Diabetes Care 22: 1462-1470, 1999.

11. Stumvoll M, Mitrakou A, Pimenta W, Jenssen T, Yki-Järvinen H, Van Haeften T, Renn W and Gerich J: Use of the oral glucose tolerance test to assess insulin release and insulin sensitivity. Diabetes Care 23: 295-301, 2000.

12. Matthews DR, Hosker JP, Rudenski AS, Naylor BA, Treacher DF and Turner RC: Homeostasis model assessment: Insulin resistance and beta-cell function from fasting plasma glucose and insulin concentrations in man. Diabetologia 28: 412-419, 1985.

13. Stancáková A, Javorský M, Kuulasmaa T, Haffner SM, Kuusisto J and Laakso M: Changes in insulin sensitivity and insulin release in relation to glycemia and glucose tolerance in 6,414 Finnish men. Diabetes 58: 1212-1221, 2009.

14. Watt KDS, Pedersen RA, Kremers WK, Heimbach JK and Charlton MR: Evolution of causes and risk factors for mortality post-liver transplant: Results of the NIDDK long-term follow-up study. Am J Transplant 10: 1420-1427, 2010.

15. Yoshizumi T, Shirabe K, Taketomi A, Uchiyama H, Harada N, Ijichi H, Yoshimatsu M, Ikegami T, Soejima Y and Maehara Y: Risk factors that increase mortality after living donor liver transplantation. Transplantation 93: 93-98, 2012.

16. Dare AJ, Plank LD, Phillips ARJ, Gane EJ, Harrison B, Orr D, Jiang Y and Bartlett AS: Additive effect of pretransplant obesity, diabetes, and cardiovascular risk factors on outcomes after liver transplantation. Liver Transpl 20: 281-290, 2014. 
17. Zein NN, Abdulkarim AS, Wiesner RH, Egan KS and Persing DH: Prevalence of diabetes mellitus in patients with end-stage liver cirrhosis due to hepatitis $\mathrm{C}$, alcohol, or cholestatic disease. J Hepatol 32: 209-217, 2000.

18. Yong CM, Sharma M, Ochoa V, Abnousi F, Roberts J, Bass NM, Niemann CU, Shiboski S, Prasad M, Tavakol M, et al: Multivessel coronary artery disease predicts mortality, length of stay, and pressor requirements after liver transplantation. Liver Transpl 16: 1242-1248, 2010.

19. Siegel AB, Lim EA, Wang S, Brubaker W, Rodriguez RD, Goyal A, Jacobson JS, Hershman DL, Verna EC, Zaretsky J, et al: Diabetes, body mass index, and outcomes in hepatocellular carcinoma patients undergoing liver transplantation. Transplantation 94: 539-543, 2012.

20. Defronzo RA: Banting Lecture. From the triumvirate to the ominous octet: A new paradigm for the treatment of type 2 diabetes mellitus. Diabetes 58: 773-795, 2009.

21. Henchoz E, D'Alessio DA, Gillet M, Halkic N, Matzinger O, Goy JJ, Chioléro R, Tappy L and Schneiter P: Impaired insulin response after oral but not intravenous glucose in heart- and liver-transplant recipients. Transplantation 76: 923-929, 2003.

22. Stockmann M, Konrad T, Nolting S, Hünerbein D, Wernecke KD, Döbling H, Steinmüller T and Neuhaus P: Major influence of liver function itself but not of immunosuppression determines glucose tolerance after living-donor liver transplantation. Liver Transpl 12: 535-543, 2006.
23. Watt KD, Dierkhising R, Fan C, Heimbach JK, Tillman H, Goldstein D, Thompson A, Krishnan A and Charlton MR: Investigation of PNPLA3 and IL28B genotypes on diabetes and obesity after liver transplantation: Insight into mechanisms of disease. Am J Transplant 13: 2450-2457, 2013.

24. Ling Q, Xie H, Lu D, Wei X, Gao F, Zhou L, Xu X and Zheng S: Association between donor and recipient TCF7L2 gene polymorphisms and the risk of new-onset diabetes mellitus after liver transplantation in a Han Chinese population. J Hepatol 58: 271-277, 2013.

25. Johansson LE, Lindblad U, Larsson CA, Råstam L and Ridderstråle M: Polymorphisms in the adiponutrin gene are associated with increased insulin secretion and obesity. Eur J Endocrinol 159: 577-583, 2008.

26. Iwata M, Maeda S, Kamura Y, Takano A, Kato H, Murakami S, Higuchi K, Takahashi A, Fujita H, Hara K, et al: Genetic risk score constructed using 14 susceptibility alleles for type 2 diabetes is associated with the early onset of diabetes and may predict the future requirement of insulin injections among Japanese individuals. Diabetes Care 35: 1763-1770, 2012.

27. Ling Q, Dong F, Geng L, Liu Z, Xie H, Xu X and Zheng S: Impacts of TCF7L2 gene polymorphisms on the susceptibility of hepatogenous diabetes and hepatocellular carcinoma in cirrhotic patients. Gene 522: 214-218, 2013. 